\title{
ON CHOOSING GENERATING SETS FOR IDEALS
}

\author{
BUDH NASHIER
}

\begin{abstract}
For an ideal $I$ in a ring $R$ the choice of a generating set with some interesting properties depends on how $I$ sits inside $R$.
\end{abstract}

1. Preliminaries. Let $R$ denote a commutative Noetherian ring with identity. For a finitely generated $R$-module $M$ over $R$, let $\mu(M)$ denote the least number of elements in $M$ required to generate $M$ as an $R$-module. If $I$ is an ideal in $R$ then $\mu\left(I / I^{2}\right)$ and $\mu(I)$ are closely related. To be precise we have the following folklore result.

Proposition. Let I be an ideal in $R$. Then $\mu\left(I / I^{2}\right) \leqslant \mu(I) \leqslant \mu\left(I / I^{2}\right)+1$.

Proof. The first inequality is trivial. For the second one, let $\mu\left(I / I^{2}\right)=n$. Choose elements $a_{1}, a_{2}, \ldots, a_{n}$ in $I$ such that $\bar{a}_{1}, \bar{a}_{2}, \ldots, \bar{a}_{n}$ generate $I / I^{2}$. Let $J=$ $\left(a_{1}, a_{2}, \ldots, a_{n}\right)$. Then $I=J+I^{2}$. Let $S=1+I$. Then $S^{-1} I=S^{-1} J+$ $\left(S^{-1} I\right)\left(S^{-1} I\right)$. Note that $S^{-1} I$ lies in the Jacobson radical of $S^{-1} R$. Hence $S^{-1} I=$ $S^{-1} J$ by Nakayama's lemma. We can find a single element $s$ in $S$ such that $s I \subset J$. If $s=1+a, a \in I$, then $I=(J, a)$. Hence $\mu(I) \leqslant n+1$.

Which one of the above inequalities becomes an equality is a question of interest. A lovely argument of Mohan Kumar [2] shows that if $\mu\left(I / I^{2}\right)>\operatorname{dim}(R)$ (Krull dimension of $R)$, then $\mu(I)=\mu\left(I / I^{2}\right)$. From the equation $I=J+I^{n}$ it follows that an extra generator for $I$, if needed, can be chosen to lie in any power of $I$. Sometimes it is possible to select a set of generators with quite interesting properties. One such situation is offered by the following theorem.

THEOREM. Let $K$ be a field and let $R$ be an affine $K$-domain of dimension $d$. Let $M$ be a regular maximal ideal of $R$ such that the residue field $L=R / M$ is a finite separable extension of $K$. Given an arbitrary nonzero element $a$ in $M^{n}$, where $n$ is an integer at least two, there exist elements $X_{1}, X_{2}, \ldots, X_{d}$ in $R$ with the following properties:

(1) $L=K\left[\bar{X}_{1}\right]$, where $\bar{X}_{1}$ denotes the residue class of $X_{1}$ modulo $M$.

(2) $M$ is generated by $f\left(X_{1}\right), X_{2}, \ldots, X_{d}$ and $a$, where $f$ denotes the minimal polynomial of $\bar{X}_{1}$ over $K$.

(3) The ring $A=R /(a)$ is integral over the polynomial ring $K\left[x_{1}, x_{2}, \ldots, x_{d-1}\right]$, where $x_{i}$ is the residue class of $X_{i}$ modulo $(a)$.

Received by the editors April 14, 1986.

1980 Mathematics Subject Classification (1985 Revision). Primary 13 F20.

(C)1987 American Mathematical Society $0002-9939 / 87 \$ 1.00+\$ .25$ per page 
2. Proof of the Theorem. Since $A$ is an affine $K$-algebra of dimension $d-1$, by Noether's Normalization Theorem [1,14.g], there exist elements $y_{1}, y_{2}, \ldots, y_{d-1}$ in $A$ such that $B=K\left[y_{1}, y_{2}, \ldots, y_{d-1}\right]$ is a polynomial ring and that $A$ is an integral ring extension of $B$. Let $\mathrm{m}=M /(a)$. Since $\mathrm{m}$ is a maximal ideal in $A, \mathfrak{m} \cap B$ is maximal in $B$. By the height consideration it follows that $(\mathfrak{m} \cap B) \cap K\left[y_{i}\right]=\mathfrak{m} \cap$ $K\left[y_{i}\right]$ is a nonzero prime ideal in $K\left[y_{i}\right]$ for $i=1,2, \ldots, d-1$. If $\mathrm{m} \cap K\left[y_{i}\right]$ is generated by the monic polynomial $g_{i}\left(y_{i}\right)$ for each $i=1,2, \ldots, d-1$, then, replacing the $y_{i}$ 's by the $g_{i}$ 's, we assume that $A$ is integral over $K\left[y_{1}, y_{2}, \ldots, y_{d-1}\right]$ and that

$$
\mathrm{m} \cap K\left[y_{1}, y_{2}, \ldots, y_{d-1}\right]=\left(y_{1}, y_{2}, \ldots, y_{d-1}\right) .
$$

Since $L$ is a finite separable extension of $K$, it is a simple extension. Let $L=R / M=K(\bar{\alpha})$, where $\alpha \in R$. Let $f$ denote the minimal polynomial of $\bar{\alpha}$ over $K$. Since $L$ is a separable extension of $K, f^{\prime}(\alpha) \notin M\left(f^{\prime}\right.$ denotes the derivative of $\left.f\right)$. If $f(\alpha) \in M^{2}$, then $f(\alpha+t) \notin M^{2}$ for any $t \in M \backslash M^{2}$. Hence we may replace $\alpha$ by $\alpha+t$, if need be, and assume that $f(\alpha) \notin M^{2}$. Thus $f(\alpha)$ is part of a minimal basis for $M\left(\bmod M^{2}\right)$.

If $z_{1}$ denotes the residue class of $\alpha$ in $A$ then $f\left(z_{1}\right) \in \mathfrak{m} \backslash \mathrm{m}^{2}$, because $a \in M^{2}$. Hence $f\left(z_{1}\right)$ is part of a minimal basis for $m\left(\bmod m^{2}\right)$. Set $x_{1}=z_{1}+y_{1}^{t_{1}}$ where $t_{1}$ is an integer at least 2. As $z_{1}=x_{1}-y_{1}^{t_{1}}$ is integral over $K\left[y_{1}, y_{2}, \ldots, y_{d-1}\right]$, by taking a sufficiently large value for $t_{1}$, it is easily checked that $y_{1}$ is integral over $K\left[x_{1}, y_{2}, \ldots, y_{d-1}\right]$. Hence $A$ is integral over $K\left[x_{1}, y_{2}, \ldots, y_{d-1}\right]$ and $f\left(x_{1}\right)$ is a part of a minimal basis for $\mathfrak{m}\left(\bmod \mathfrak{m}^{2}\right)$.

Now we choose $z_{2} \in A$ such that $f\left(x_{1}\right)$ and $z_{2}$ are part of a minimal basis for $m$ $\left(\bmod \mathrm{m}^{2}\right)$. Set $x_{2}=z_{2}+y_{2}^{t_{2}}$ where $t_{2}$ is an integer at least 2 . Then by taking a large value of $t_{2}$, as done above, we conclude that $A$ is integral over $K\left[x_{1}, x_{2}, y_{3}, \ldots, y_{d-1}\right]$ and $f\left(x_{1}\right), x_{2}$ are part of a minimal basis for $\mathrm{m}\left(\bmod \mathrm{m}^{2}\right)$. We continue replacing the $y_{i}$ 's by $x_{i}$ 's and eventually obtain elements $x_{1}, x_{2}, \ldots, x_{d-1}$ in $A$ such that $A$ is integral over $K\left[x_{1}, x_{2}, \ldots, x_{d-1}\right]$ and that $f\left(x_{1}\right), x_{2}, \ldots, x_{d-1}$ are part of a minimal basis for $m\left(\bmod m^{2}\right)$.

Note that $\mu\left(\mathrm{m} / \mathrm{m}^{2}\right)=\mu\left(M / M^{2}\right)$ as $a \in M^{2}$. Since $M$ is a regular maximal ideal of $R$, we have $\mu\left(M / M^{2}\right)\left(=\mu\left(M R_{M}\right)\right)=\operatorname{dim}\left(R_{M}\right)=\operatorname{dim}(R)=d$. Let $I$ be the ideal in $A$ generated by $f\left(x_{1}\right), x_{2}, \ldots, x_{d-1}$. Let $C=A / I$ and $\mathfrak{n}=\mathfrak{m} / I$. Then $C$ is a zero-dimensional ring (being integral over a field isomorphic to $L$ ). Note that $\mu\left(\mathfrak{n} / \mathfrak{n}^{2}\right)=\mu\left(\mathrm{m} / \mathrm{m}^{2}\right)-(d-1)=1$. Hence $\mu(\mathfrak{n})=1$. We choose $x_{d}$ in $A$ such that $\mathrm{m}=\left(I, x_{d}\right)$. If $X_{1}, X_{2}, \ldots, X_{d}$ are lifts in $R$ of the elements $x_{1}, x_{2}, \ldots, x_{d}$ respectively, then the properties (1), (2), and (3) are satisfied. Thus the proof is complete.

Examples of rings as in the above theorem are readily available in algebraic geometry. The coordinate ring of a smooth irreducible algebraic variety over a perfect field enjoys this property.

\section{REFERENCES}

1. H. Matsumura, Commutative algebra (2nd ed.), Benjamin, New York, 1980.

2. N. Mohan Kumar, On two conjectures about polynomial rings, Invent. Math. 46 (1978), 225-236.

Department of Mathematics, Florida State University, Tallahassee, Florida 32306 - 3027 